\title{
Abstracts presented at the Laryngology and Rhinology Section Meetings, Royal Society of Medicine, 4 March 2016, London, UK
}

\author{
Anti-tumour necrosis factor therapy is a risk factor for \\ certain subtypes of chronic rhinosinusitis \\ C G Leonard, C Masih, S McDonald, G Taylor, N Maiden, \\ P J Leyden \\ From the Craigavon Area Hospital, Northern Ireland

\section{Background}

There are many risk factors for chronic rhinosinusitis; however, the effect of anti-tumour necrosis factor (antiTNF) therapy has not been investigated in depth. Our experience points to a detrimental clinical effect in overall prevalence of chronic rhinosinusitis, despite its benefit in certain subtypes.

\section{Methods}

A telephone survey was performed to parallel the findings of the Global Allergy and Asthma European Network ('GA2LEN') chronic rhinosinusitis screening survey. This was itself based on the widely recognised European Position Paper on Rhinosinusitis and Nasal Polyps ('EP3OS') criteria.

\section{Results}

A total of 120 patients responded to the survey. The prevalence of chronic rhinosinusitis in the sample (anti-TNF therapy) population was 20 per cent ( 95 per cent confidence interval $=12.84-27.16$ ). Anti-TNF therapy is therefore a risk factor for chronic rhinosinusitis when compared, using a one-sample test of proportions, with prevalence in the wider population.

\section{Conclusion}

This is the first observational study indicating increased prevalence of chronic rhinosinusitis in patients treated with anti-TNF therapy. These clinical findings require investigation in greater depth to clarify the nature of pathologies we currently diagnose and treat as chronic rhinosinusitis.

Transoral partial oropharyngectomy for oropharyngeal cancer

R Gan, M Nistor, G Wijayasingam, S Mortimore, M De From the Royal Derby Hospital

\section{Introduction}

Transoral surgery has gained popularity as a treatment of oropharyngeal cancer (Sheahan, 2015). This study aimed to analyse the oncological and survival outcomes of patients who had undergone transoral surgery for oropharyngeal cancer.

\section{Methods}

A retrospective study was conducted of oropharyngeal cancer patients treated with primary transoral partial oropharyngectomy between 29 August 2001 and 13 October 2015 performed by two consultants.

Results

Thirty-one patients were included. Seven (22.6 per cent) had stage I disease, 3 (9.7 per cent) stage II, 6 (19.4 per cent) stage III and 15 (48.4 per cent) stage IV. Twelve patients (38.7 per cent) were human papillomavirus positive. Twenty-five patients were treated with transoral laser surgery with or without neck dissection and/or chemoradiotherapy. Six patients had transoral robotic surgery with or without neck dissection and/or chemoradiotherapy. Five patients (16.1 per cent) had disease recurrence. Six patients (19.4 per cent) had positive margins on resection. The fiveyear disease-free survival rate was 78 per cent, and the disease-specific survival rates at two and five years were both 85 per cent. Five patients (16.1 per cent) died during follow up.

\section{Conclusion}

Transoral partial oropharyngectomy is a valid treatment in a selected group of patients with oropharyngeal cancer.

Altered transforming growth factor beta signalling in inflammatory nasal polyps drives remodelling in chronic rhinosinusitis with nasal polyps

N T Orban, A Eifan, M Jacobson, S Durham

From the Imperial College London

\section{Introduction}

Dysregulation of transforming growth factor beta and activin signalling play fundamental roles in lower airways remodelling, with unknown relevance in the nose.

\section{Methods}

Immunohistochemical staining was performed on inferior turbinates and nasal polyps. The following variables were measured in chronic rhinosinusitis with nasal polyps participants $(n=10)$ and in healthy controls $(n=19)$ : transforming growth factor beta 1; activin-A, its receptor ALK-4 and pSMAD2; D2-40 and cluster of differentiation 34 (lymphatic and vascular remodelling); smooth muscle actin and heat shock protein 47 (collagen synthesis and myofibroblast transformation); and matrix metalloproteinases 7 and 9, with their inhibitor tissue inhibitor of metalloproteinases 1 .

Results

Turbinates showed increased lymphatic and vascular remodelling, with polyps nearly devoid of glands and possessing 
very little blood vessels. Heat shock protein 47 , matrix metalloproteinases 7 and 9, and tissue inhibitor of metalloproteinases 1 expression was elevated in polyps; smooth muscle actin was increased in turbinates. Transforming growth factor beta 1 expression was increased in polyps, with ALK-4 elevated in polyps and turbinates. Activin levels tended to be higher in polyps of chronic rhinosinusitis with nasal polyps patients.

\section{Discussion}

These data demonstrate remodelling alterations in polyps and turbinates of chronic rhinosinusitis with nasal polyps cases, with dysregulated transforming growth factor beta 1 signalling in inflammatory polyps driving chronic changes in tissue architecture.

A long-term prospective evaluation comparing roboticassisted thyroidectomy with open thyroidectomy in a UK population

G Garas, A Arora, S Sharma, F Palazzo, A Darzi, N Tolley

From the St Mary's Hospital, Imperial College London

\section{Objectives}

Robotic-assisted thyroidectomy was pioneered in South Korea, where cultural factors, anthropometry and remuneration favour this approach. Small thyroid nodules account for the majority of cases here because of a national thyroid cancer screening programme. The objective was to evaluate robotic-assisted thyroidectomy in the UK, where patients with larger thyroid nodules tend to undergo surgery, in a population with a significantly higher body mass index.

\section{Methods}

A prospective, non-randomised study was conducted. Sixteen consecutive robotic-assisted thyroidectomy patients were compared to 16 open thyroidectomy controls; all procedures were performed by the same team, in a tertiary referral endocrine surgery centre.

\section{Results}

There was no need for conversion to open surgery, and no significant differences regarding pain, voice, swallowing, quality of life, blood loss or scar length. In the roboticassisted thyroidectomy group, there was one case of recurrent laryngeal nerve paresis and one case of temporary shoulder dysfunction. Both resolved within four weeks. Scar cosmesis was superior following robotic-assisted thyroidectomy, even after three years $(p=0.02)$, but operative time was significantly longer in this group (228 minutes vs 85 minutes, $p=0.01$ ).

\section{Conclusion}

Robotic-assisted thyroidectomy is safe and feasible in a selected UK population, achieving superior cosmesis at the expense of time and cost.

Hypopharyngeal cancer before and during the epoch of organ preservation: a 30-year experience

R Lawrence, D G Grant, S Koonce, S Bathala, R Alibone, P J Bradley

Queen's Medical Centre, Nottingham

\section{Background}

The introduction of non-surgical organ-preserving treatment protocols in the late 1990s has led to a transformation in attitudes toward the surgical management of head and neck cancer.

\section{Methods}

A 32-year retrospective review of 288 patients with cancer of the hypopharynx was conducted to identify surgical and oncological outcomes and treatment choices prior to and during the epoch of organ preservation.

Results

Between 1983 and 2000, 33 per cent of patients were treated with primary radiotherapy (RT), and 34 per cent were treated with surgery with or without adjuvant RT. After 2000, 17 per cent of patients received chemoradiotherapy, 26 per cent received RT, and 43 per cent underwent surgery with or without adjuvant RT. The overall five-year survival rates for surgical and non-surgical management prior to 2000 were 28 per cent and 15 per cent respectively. Following 2000 , the surgical and non-surgical overall survival rates were 20 per cent and 26 per cent respectively $(p>0.05)$

\section{Conclusion}

The proportion of patients receiving surgical and non-surgical treatment remains largely unchanged. Survival rates remain disappointingly static. A detailed analysis was conducted. 